\title{
Impact of operator experience and wiring technique on procedural efficacy of trans-radial percutaneous chronic total occlusion recanalization performed by dedicated radialists
}

Francesco Burzotta, Carlo Trani, Antonella Tommasino, Marta Francesca Brancati, Silvia Saffioti, Giancarlo Pirozzolo, Giampaolo Niccoli, Antonio Maria Leone, Giovanni Schiavoni, Filippo Crea

Institute of Cardiology, Catholic University of Sacred Heart, Rome, Italy

\begin{abstract}
Background: The efficacy of trans-radial approach (TRA) in chronic total occlusions (CTO) percutaneous coronary interventions (PCI) is not well established. Thus, we sought to review the feasibility and long-term results of TRA for CTO PCI performed by dedicated TRA operators of our center.
\end{abstract}

Methods: CTO PCI performed by dedicated radialists were considered. Primary end-points were "PCI success" (stent implantation with residual stenosis $<20 \%$ and TIMI 3) and "patient success" (PCI success in a first or second attempt). Vascular complications and major adverse cardiac events (MACE) were also assessed. Procedures were divided into: Period 1 no systematic adoption of TRA nor systematic wire selection, and Period 2 - systematic TRA with stepwise wire selection. The starting guidewire was initially an intermediate wire (Period $2 a$ ), and, thereafter, a tapered soft polymeric guidewire (Period $2 b$ ).

Results: Two operators performed 167 TRA PCI on CTO in 158 patients. PCI success rate was $74.3 \%$ and patient success rate was $78.5 \%$. Drug-eluting stents were implanted in $95.1 \%$ of successful procedures. One (0.6\%) patient had a (minor) vascular complication. After a mean follow-up of 580 days, $93.7 \%$ of patients were free from MACE. PCI success $(57.1 \%$ in Period 1 vs. $76.5 \%$ in Period $2 a$ vs. $80.5 \%$ in Period $2 b, p=0.029$ ) and patient success (62.5\% in Period 1 vs. $77.8 \%$ in Period $2 a$ vs. $86.1 \%$ in Period $2 b, p=0.025$ ) significantly improved during the study.

Conclusions: CTO PCI by TRA is safe and feasible. Its efficacy seems to be strongly dependent on operator experience with CTO techniques and may be influenced by the strategy of guidewire selection. (Cardiol J 2013; 20, 5: 560-567)

Key words: radial approach, percutaneous coronary intervention, chronically occluded vessels, procedural efficacy, operator experience, wiring technique

Address for correspondence: Francesco Burzotta, MD, PhD, Institute of Cardiology, Catholic University of Sacred Heart, L.go Gemelli 1, 00168 Rome, Italy, tel: +39 3494295290, fax: + 3906 3055535, e-mail: f.burzotta@rm.unicatt.it 


\section{Introduction}

Percutaneous coronary intervention (PCI) in chronic total occlusion (CTO) represents an evolving field of interventional cardiology. Since complex techniques and high support are often required, large guiding catheters inserted by trans-femoral approach are usually adopted by CTO-dedicated operators [1]. As a consequence, the majority of interventionalists who perform CTO PCI consider trans-radial approach (TRA) unsuitable for such kind of interventions. On the opposite, some dedicated trans-radial operators use to select TRA for CTO PCI. Operator's experience with both TRA and CTO interventions is probably important for the safety and efficacy of TRA CTO. In the present study, we assessed the acute and long-term results of consecutive TRA CTO PCI performed by dedicated radial operators. Moreover, we sought to assess the impact of operator's experience and wiring technique on procedural efficacy.

\section{Methods}

From January 2006 to May 2011, consecutive patients admitted to our Institution, undergoing the TRA as a first access, were screened to enter the present registry. Among those, $\mathrm{PCI}$ on CTO lesions performed by operators having a $>90 \%$ TRA rate at study beginning ("dedicated radialists" [2]) were included in the present retrospective study.

CTO lesions were defined as TIMI 0 flow within the occluded segment with an estimated occlusion duration of $>3$ months [1].

Demographic, clinical and procedural data were prospectively collected on a dedicated database agreed on by all the operators of the center. Recorded procedural data included the need for access crossover defined as failure to successfully complete the procedure by the first attempted approach.

Patients with renal failure requiring dialysis were not considered for the radial or ulnar approach in order to avoid any damage to the forearm circulation. The Allen test was routinely used to screen the patient suitable for radial artery catheterization. In the case of abnormal Allen test, the Barbeau test was performed.

Patients gave written informed consent to the procedure.

\section{Trans-radial PCI technique adopted during all the study}

An antegrade approach through 6 French guiding catheter was the main revascularization strategy adopted. Larger (7-8 French) lumen guiding catheters were used in a minority of patients who required an intravascular ultrasound-guided CTO proximal cap puncture. Systematic double radial approach was selected when distal vessel was not visualized by intracoronary collateral circulation. Figure 1 shows the cath lab set up at our center for a patient undergoing CTO PCI using bi-radial approach. As a general rule, the left radial approach was selected for PCI in occluded right coronary CTO while right radial approach was used for CTO located in the left coronary artery in order to gain the maximal guiding catheter support [3]. After successful lesion crossing with the guidewire, multiple balloon dilations were performed. Drug-eluting stents were then used in the majority of patients. A strategy of aggressive stent post-dilation with non-compliant balloons and full lesion coverage (with multiple stents if needed) was adopted.

All patients were on double antiplatelet therapy with aspirin (100 mg/day) and clopidogrel (300 mg loading dose followed by $75 \mathrm{mg} /$ day) before PCI attempt. In the cath lab, weight-adjusted (100 IU/kg) bolus was administered through the sheath at procedure beginning and was eventually followed by further intravenous heparin boluses to maintain activated clotting time between 250 and $300 \mathrm{~s}$. IIb/IIIa inhibitors were not used. Vasodilator drugs were not routinely administered and only nitrates were used in the case of documented or suspected radial artery spasm. The arterial sheath was always withdrawn after the procedure. A bandage made of a gauze pad and 3 adhesive elastic bands was used for compression. The bandage was slackened after $1 \mathrm{~h}$ and kept on for 4-6 h after procedures.

\section{Specific CTO wiring techniques used during various phases of the study}

Although the above described general set up for PCI attempt was maintained throughout the study, a significant evolution in the specific approach for CTO lesion crossing was observed during the study. Such evolution mainly reflected a change in the strategy of wire selection. Indeed, we firstly introduced in our practice the teaching received by a Japanese expert CTO operator who recommended a specific selection of step-wise increase in guidewire stiffness and, later on, we started using a novel tapered, soft hydrophilic guidewire. During these phases, also the confidence in selecting the radial approach for CTO increased.

Such evolution allowed us to divide the study into 3 sequential phases as follows:

- Period 1 (first part of the study): no systematic adoption of radial access, no systematic wiring technique was applied. During this phase, the 


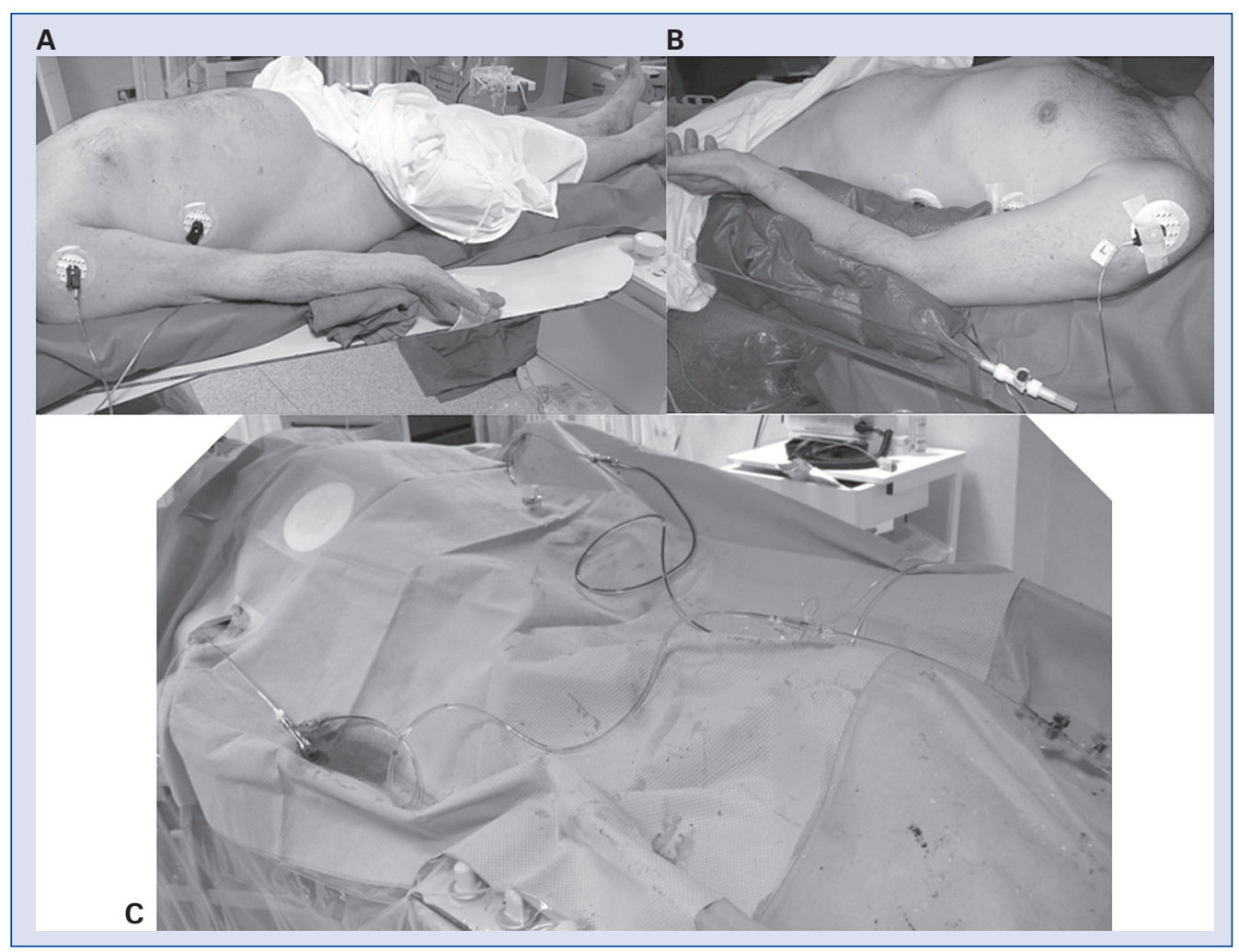

Figure 1. Patient set up for chronic total occlusions recanalization requiring bi-radial approach; $\mathbf{A}$. Set up of right arm; B. Set up of left arm using a pneumatic pillow; C. Appearance of the operative table during intervention (a dedicated sterile drape with left and right radial holes is used for bi-radial approach).

operator decided to use the TRA in selected cases (cases requiring double injection were usually excluded) and the selection of guidewires was performed according to the anatomical yield of CTO lesion;

- Period 2 (second part of the study): TRA was systematically adopted and a systematic stepwise wire selection was applied. During the former Period 2a, the wire sequence was: intermediate (Abbott Vascular, USA), Miracle 3 (Asahi Intecc, Aichi, Japan), Miracle 6 (Asahi Intecc, Aichi, Japan), Confianza Pro (Asahi Intecc, Aichi, Japan). During the following Period 2b, the first wire (instead of Intermediate wire) was the Fielder XT (Asahi Intecc, Aichi, Japan), a tapered hydrophilic ("sliding" technique); the remaining wire selection steps were identical to those in Period 2a. The study design is represented in Figure 2.

\section{Study end-points}

Study end-points were the following:

- "PCI success" (successful stent implantation with visual residual stenosis $<20 \%$ and TIMI flow grade 3);
- "Patient success" (PCI success in the first or second attempt).

Vascular complications and clinical outcome After PCI, the in-hospital clinical course was carefully monitored, whereas, after discharge, patients were followed-up by telephone or hospital visit at $9,12,24$, and 36 months.

During hospitalization, the occurrence of a possible access-site vascular complication was prospectively recorded in the database after its suspected arising. Patients with suspected vascular complication underwent an instrumental investigation which, according to our standard protocol, included peripheral angiography if the suspect was intra-procedural and ultrasound evaluation if the suspect was post-procedural. Other types of instrumental investigations were eventually performed if deemed necessary. Vascular complications were defined as any vascular damage documented by specific instrumental investigations [2, 4].

Major adverse cardiac events (MACE), including cardiac death, non fatal myocardial infarction (MI), stent thrombosis and target vessel revascularization (TVR) were assessed. All deaths of 


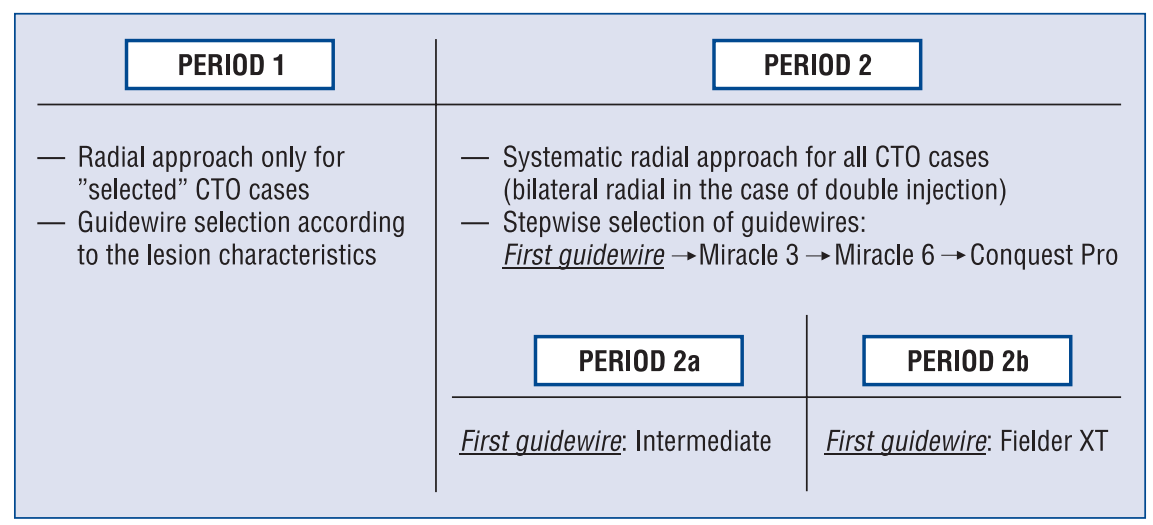

Figure 2. Study design; CTO - chronic total occlusion.

unknown cause were considered of cardiovascular origin. MI included post-procedural MI and MI during clinical follow-up. TVR was defined as repeat clinically driven revascularization by either PCI or coronary artery bypass grafting involving the treated vessel. Stent thrombosis was classified by the Academic Research Consortium as definite, probable, or possible, and as early ( 0 to 30 days), late (31 to 360 days), or very late (over 360 days) [5].

\section{Statistical analysis}

Continuous variables were checked for normality using the Kolmogorov-Smirnov test, presented as mean \pm standard deviation, and compared using the Student's unpaired t-test. Categorical variables are presented as counts and percentages, and compared by means of $\chi^{2}$ tests or Fisher exact test, as appropriate. A two tailed, $\mathrm{p}$ value $<0.05$ was established as the level of statistical significance for all tests.

\section{Results}

At the beginning of the study period, 2 operators of our institution had $>90 \%$ TRA rate. These two operators attempted 167 TRA PCI on CTO in 158 patients. Of note, most of the procedures were conducted with both operators scrubbed at the operative table.

The characteristics of the study population are reported in Table 1 . Briefly, mean age was 64 years, $69.6 \%$ were stable, $61.2 \%$ had multivessel disease. The PCI procedure was attempted by either a single radial approach (in the case of visualization of collateral circulation by collateral circulation coming from same coronary artery) or by double radial approach (when collateral circulation came only from a different native or graft vessel: 57 cases, $34.1 \%$ ). Actually, only in 2 cases the operator failed to complete the procedure by full trans-radial approach (access crossover rate: $1.2 \%, 2 / 167$ procedures): both cases started with bi-radial approach and the femoral access (using $5 \mathrm{~F}$ diagnostic equipment) was adopted to visualize the collateral circulation after one-side radial access failure. Neither of the cases was encountered in the last study period (Period $2 \mathrm{~b}$ ) and both were due to the failure to catheterize a right radial artery (previously used for multiple coronary procedures) and to cross a radial artery loop.

Technical details of PCI procedures are reported in Table 2. PCI success was achieved in 124 procedures leading to a PCI success rate of $74.3 \%$ and patient success rate of $78.5 \%$. Drug eluting stents were implanted in $95.1 \%$ of successful procedures (Table 2 ). One $(0.6 \%)$ patient only had an angiographic complication (minor guidewire-induced coronary perforation sealed by anticoagulation reversal and prolonged balloon inflation). No catheter-induced iatrogenic dissection of coronary ostia occurred. A minor access-site vascular complication occurred in $1(0.6 \%)$ patient who had a forearm hematoma which was successfully managed by prolonged compressive bandage without any need of blood transfusion nor surgery.

After a mean follow-up time of $580 \pm 305$ (range 270-1080) days, 93.7\% of patients were free from MACE. A detailed report of adverse events occurring during the study is provided in Table 3.

During the study, despite a growing complexity of attempted lesions after Period 1 (Table 2), a significant improvement in the overall efficacy of the procedure was observed. In particular, PCI success increased from $57.1 \%$ in Period 1 to $78.8 \%$ in Period $2(\mathrm{p}=0.01)$ (Table 2$)$ while patient success increased from $62.5 \%$ in Period 1 to $82.5 \%$ $(\mathrm{p}=0.01)$. Interestingly, as shown in Figure 3, 
Table 1. Main clinical characteristics of the study population and of patients belonging to Period 1 and Period 2.

\begin{tabular}{|c|c|c|c|c|}
\hline & $\begin{array}{c}\text { All } \\
(n=158)\end{array}$ & $\begin{array}{l}\text { Period } 1 \\
(n=32)\end{array}$ & $\begin{array}{l}\text { Period } 2 \\
(n=126)\end{array}$ & $\begin{array}{c}P \\
\text { (1 vs. 2) }\end{array}$ \\
\hline Age & $65 \pm 10$ & $64 \pm 10$ & $65 \pm 10$ & 0.8 \\
\hline Male gender & $139(87.9 \%)$ & $26(81.2 \%)$ & $113(89.7 \%)$ & 0.2 \\
\hline \multicolumn{5}{|l|}{ Risk factors: } \\
\hline Family history of IHD & $53(33.5 \%)$ & $14(43.7 \%)$ & $39(30.9 \%)$ & 0.2 \\
\hline Diabetes mellitus & $47(29.7 \%)$ & $9(28.1 \%)$ & $38(30.1 \%)$ & 1.0 \\
\hline Hypertension & $122(77.2 \%)$ & $21(65.6 \%)$ & $101(80.1 \%)$ & 0.1 \\
\hline Hypercholesterolemia & $113(71.5 \%)$ & $20(62.5 \%)$ & $93(73.8 \%)$ & 0.3 \\
\hline Active smoking & $22(13.9 \%)$ & $7(21.9 \%)$ & $15(11.9 \%)$ & 0.1 \\
\hline Renal failure & $21(13.2)$ & $4(12.5 \%)$ & $17(13.5 \%)$ & 1.0 \\
\hline \multicolumn{5}{|l|}{ Clinical history: } \\
\hline Stable angina/silent ischemia & $104(65.8 \%)$ & $19(59.4 \%)$ & $85(67.4 \%)$ & 0.4 \\
\hline Recent ( $>48 \mathrm{~h} \leq 3$ months) NSTE-ACS & $18(11.4 \%)$ & $4(12.5 \%)$ & $14(11.1 \%)$ & 0.8 \\
\hline Recent (> $48 \mathrm{~h} \leq 3$ months) STEMI & $4(2.5 \%)$ & $1(3.1 \%)$ & $3(2.4 \%)$ & 1.0 \\
\hline Previous MI (> 3 months) & $42(26.6 \%)$ & $8(25.0 \%)$ & $34(26.9 \%)$ & 0.7 \\
\hline Previous $\mathrm{PCl}$ & $54(34.2 \%)$ & $10(31.2 \%)$ & $44(34.9 \%)$ & 0.8 \\
\hline Previous coronary surgery & $27(17.1 \%)$ & $4(12.5 \%)$ & $23(18.2 \%)$ & 0.6 \\
\hline Impaired LV function (EF < 50\%) & $38(24.0 \%)$ & $9(28.1 \%)$ & $29(23.0 \%)$ & 0.6 \\
\hline \multicolumn{5}{|l|}{ Extent of coronary artery disease: } \\
\hline Single-vessel disease & $64(40.5 \%)$ & $13(40.6 \%)$ & $51(40.5 \%)$ & 1.0 \\
\hline Two-vessel disease & $42(26.6 \%)$ & $11(34.4 \%)$ & $31(24.6 \%)$ & 0.3 \\
\hline Three-vessel disease & $52(32.9 \%)$ & $8(25.0 \%)$ & $44(34.9 \%)$ & 0.4 \\
\hline Left main disease & $8(5.1 \%)$ & $0(0 \%)$ & $8(6.3 \%)$ & 0.4 \\
\hline
\end{tabular}

IHD — ischemic heart disease; STEMI - ST-elevation myocardial infarction; NSTE-ACS — non-ST-elevation acute coronary syndrome; $\mathrm{MI}$ - myocardial infarction; $\mathrm{PCl}$ - percutaneous coronary intervention; LV — left ventricular; EF — ejection fraction

a progressive increase in procedure efficacy was noted since PCI success significantly improved during the 3 study periods: $57.1 \%$ in Period 1 vs. $76.5 \%$ in Period 2a vs. $80.5 \%$ in Period $2 \mathrm{~b}$ $(\mathrm{p}=0.029)$. Similar improvement was observed for patients success: $62.5 \%$ in Period 1 vs. $77.8 \%$ in Period 2a vs. $86.1 \%$ in Period $2 \mathrm{~b}(\mathrm{p}=0.025)$. The positive impact of "sliding" technique based on Fielde XT wire on procedure efficacy is supported by Figure 4, which shows the rate of successful lesion crossing with the first wire across the 3 study periods.

\section{Discussion}

CTOs represent the most technically challenging lesions for PCI and, according to the standard practice of the majority of CTO-dedicated centers and operators, PCI on CTO are attempted using large guiding catheters and trans-femoral approach. In the present manuscript we show that:

- TRA for PCI on CTO is feasible for dedicated radial operators;
- efficacy of TRA for CTO PCI is characterized by a learning curve and appears to be favorably influenced by the adoption of "sliding" wiring technique based on a last generation tapered hydrophilic wire.

TRA is a valuable alternative to TFA associated with a reduction of vascular complications $[6,7]$ and possibly to a better clinical outcome especially in acute coronary syndromes [8]. Thus, the use of TRA for routine PCI is rapidly expanding while its application to complex procedures is under evaluation.

In the specific field of CTO PCI, usually, the need for high back up force to advance devices into the coronary tree and the possible need for complex techniques requiring simultaneously multiple devices has traditionally lead to the use of large guiding catheters through the femoral access as a default strategy in the majority of cath labs [9]. Yet, progressive miniaturization of materials and evolution of wiring techniques may currently allow to tackle the majority of CTO by $6 \mathrm{~F}$ guiding catheters [4]. Moreover, in the hand of expert TRA operators, 
Table 2. Angiographic and procedural characteristics of the study population and of procedures conducted in Period 1 and Period 2.

\begin{tabular}{|c|c|c|c|c|}
\hline & $\begin{array}{c}\text { All } \\
(n=167)\end{array}$ & $\begin{array}{l}\text { Period } 1 \\
(n=35)\end{array}$ & $\begin{array}{l}\text { Period } 2 \\
(n=132)\end{array}$ & $\begin{array}{c}\text { P } \\
\text { (1 vs. 2) }\end{array}$ \\
\hline \multicolumn{5}{|l|}{ Angiographic characteristics } \\
\hline \multicolumn{5}{|l|}{ CTO lesion location: } \\
\hline Left anterior descending artery & $70(41.9 \%)$ & $12(34.3 \%)$ & $58(43.9 \%)$ & 0.3 \\
\hline Left circumflex artery & $33(19.8 \%)$ & $9(25.7 \%)$ & $24(18.2 \%)$ & 0.3 \\
\hline Right coronary artery & $63(37.7 \%)$ & $14(40.0 \%)$ & $49(37.1 \%)$ & 0.8 \\
\hline \multicolumn{5}{|l|}{ CTO lesion features: } \\
\hline Micro-channels & $46(27.5 \%)$ & $8(22.8 \%)$ & $38(28.8 \%)$ & 0.5 \\
\hline "Tapered" & $45(26.9 \%)$ & $8(22.8 \%)$ & $37(28.0 \%)$ & 0.7 \\
\hline "Stump"/not evident occlusion site & $85(50.9 \%)$ & $13(37.1 \%)$ & $72(53.8 \%)$ & 0.09 \\
\hline "Bridging collaterals" or "caput medusae" & $58(34.7 \%)$ & $9(25.7 \%)$ & $49(37.1 \%)$ & 0.2 \\
\hline Homocoronary (intercoronary) collaterals & $55(32.9 \%)$ & $9(25.7 \%)$ & $46(34.8 \%)$ & 0.4 \\
\hline Heterocoronary collaterals & $82(49.1 \%)$ & $10(28.6)$ & $72(54.5 \%)$ & 0.01 \\
\hline CTO in bifurcation & $41(24.5 \%)$ & $11(31.4)$ & $30(22.7 \%)$ & 0.4 \\
\hline \multicolumn{5}{|l|}{ Procedural characteristics } \\
\hline \multicolumn{5}{|l|}{ Vascular access: } \\
\hline Left radial only & $34(20.3 \%)$ & $8(22.8 \%)$ & $26(19.7 \%)$ & 0.6 \\
\hline Right radial only & $79(47.3 \%)$ & $20(57.1 \%)$ & $59(44.7 \%)$ & 0.2 \\
\hline Double radial & $51(30.5 \%)$ & $5(14.3 \%)$ & $46(34.8 \%)$ & 0.02 \\
\hline Crossover to femoral & $2(1.2 \%)$ & $1(2.8 \%)$ & $1(0.7 \%)$ & 0.4 \\
\hline \multicolumn{5}{|l|}{ PCl details: } \\
\hline Microcatheter (instead of over-the-wire balloon) & $38(22.7 \%)$ & $0(0.0 \%)$ & $38(28.8 \%)$ & $<0.01$ \\
\hline Guiding catheter larger than $6 \mathrm{~F}$ & $9(5.4 \%)$ & $1(2.8 \%)$ & $8(6.1 \%)$ & 0.7 \\
\hline Need of deep intubation & $13(7.8 \%)$ & $2(5.7 \%)$ & $11(8.3 \%)$ & 1.0 \\
\hline Anchoring balloon technique & $7(4.2 \%)$ & $0(0.0 \%)$ & $7(5.3 \%)$ & 0.3 \\
\hline IVUS-guidance for proximal cap identification & $5(2.9 \%)$ & $0(0.0 \%)$ & $5(3.8 \%)$ & 0.6 \\
\hline Debulking technique (rotablator or laser) & $6(3.6 \%)$ & $0(0.0 \%)$ & $6(4.5 \%)$ & 0.3 \\
\hline \multicolumn{5}{|l|}{ Stents: } \\
\hline Bare metal stent & $6(3.6 \%)$ & $3(8.5 \%)$ & $3(2.3 \%)$ & 0.1 \\
\hline Drug-eluting stent & $118(70.6 \%)$ & $17(48.6 \%)$ & $101(76.5 \%)$ & $<0.01$ \\
\hline Stent number & $1.8 \pm 0.9$ & $1.7 \pm 0.9$ & $1.9 \pm 0.8$ & 0.6 \\
\hline Stent size & $3.2 \pm 0.5$ & $3.0 \pm 0.5$ & $3.2 \pm 0.5$ & 0.06 \\
\hline Stent length & $49.4 \pm 23.8$ & $43.4 \pm 23.2$ & $50.5 \pm 23.8$ & 0.2 \\
\hline $\mathrm{PCl}$ success & $124(74.2 \%)$ & $20(57.1 \%)$ & $104(78.8 \%)$ & 0.01 \\
\hline
\end{tabular}

СTO - chronic total occlusion; IVUS — intravascular ultrasound; $\mathrm{PCl}$ - percutaneous coronary intervention

Table 3. Major adverse cardiac events (MACE) observed during the follow-up (hierarchical).

\begin{tabular}{lc}
\hline & Patients (n = 158) \\
\hline Any MACE & $10(6.3 \%)$ \\
Cardiac death & $3(1.9 \%)$ \\
Myocardial infarction & 0 \\
TVR & $7(4.5 \%)$ \\
Re-PCl & $4(2.5 \%)$ \\
CABG & $3(1.9 \%)$ \\
Definite stent thrombosis & 0 \\
Probable stent thrombosis & 0 \\
\hline
\end{tabular}

TVR — target vessel revascularization; CABG — coronary artery bypass graft; $\mathrm{PCl}$ - percutaneous coronary intervention bilateral radial access and appropriate guiding catheter selection and manipulation allows to have an excellent back-up from the guiding catheter [4].

In the present study we report on the experience earned in the last years by dedicated trans-radial operators operating in a high volume TRA centre [2]. The utilization of TRA CTO PCI in this specific environment has been encouraged by the observation that access crossover and coronary or peripheral complications were rare [2]. Our data suggest that in a dedicated TRA centre TRA can be considered also for highly complex PCI like CTO. The possible relevance of TRA experience in TRA CTO PCI is also suggested by the highly variable 


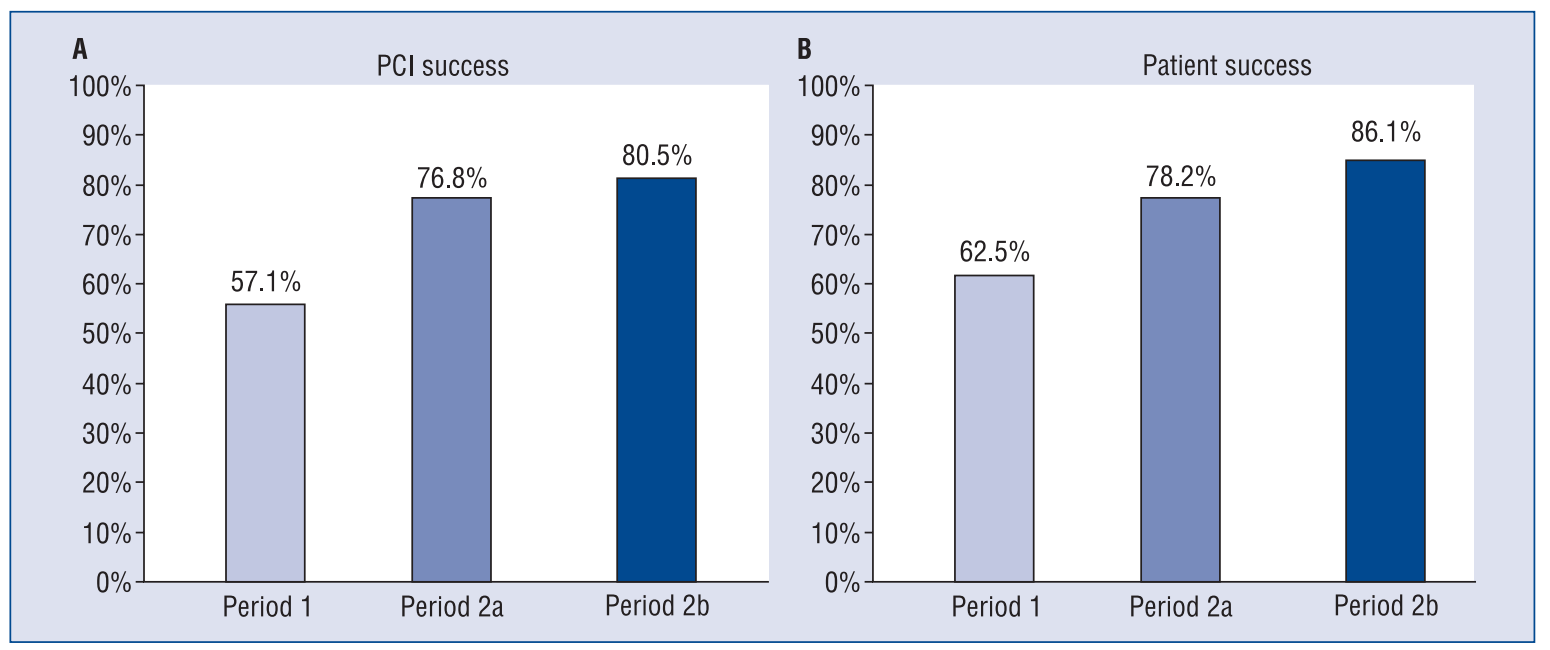

Figure 3. Procedure success and patient success during the three "wiring strategy" phases of the study; $\mathrm{PCl}$ - percutaneous coronary intervention.

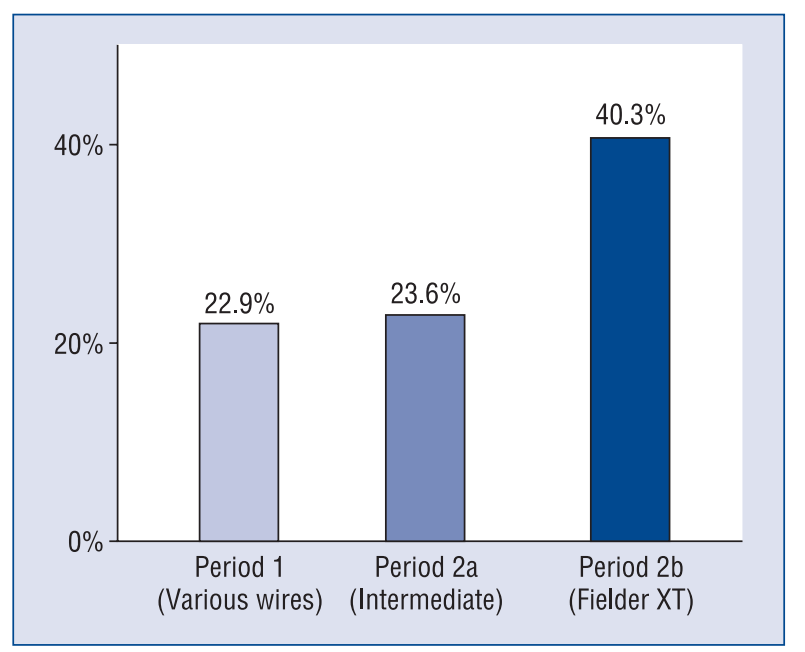

Figure 4. Rate of successful lesion crossing with the first wire during the three "wiring strategy" phases of the study.

rate of access failure reported by previously published studies [5, 10-13]. Moreover, since access crossovers are mainly due to anatomical variants of radial and subclavian arteries [11], the ability to promptly recognize and appropriately overcome upper limb anatomic variants [14] is probably pivotal to successfully complete the procedure by TRA.

Moving from procedure safety to procedure efficacy, procedure success rates should be carefully considered. In CTO PCI studies, the issue of matching between lesion complexity and operator experience with CTO are probably the main determinants of PCI success. Such concepts are strongly supported by our study which shows increasing success rates despite worse angiographic features. Similar results were observed in the specific setting of TRA CTO PCI by other authors $[5,10]$.

Trying to provide better characterization on the main evolution of our CTO technique during the study, we were able to distinguish 3 phases on the bases of guidewire selection. In the first phase, we tried to select at procedure beginning the best guidewire on the basis of lesion anatomy. Such approach may lower the number of procedures initiated by each guidewire (thus limiting the operator's learning of best manipulation of each specific guidewire) and may increase the risks of unnecessary subintimal track in the case of incorrect stiffer wire selection for relatively soft lesions with adverse angiographic aspect (since no angiographic feature may accurately predict lesion stiffness). Thereafter, we moved to a strategy of stepwise increase in guidewire stiffness, observing an impressive increase in success rates. Finally, after the availability in Europe of the Fielder XT (Asahi Intecc, Aichi, Japan), a unique guidewire characterized by hydrophilic coating and soft tapered tip, we started to use such guidewire as the very first step in order to try navigate the (often angiographically-invisible) micro-channels within the tissue of the CTO lesion ("sliding" technique). The high rates of success and low adverse events observed suggest that the approach of antegrade attempt of CTO crossing with sliding technique followed by eventual stiffer wire selections via radial approach is a safe and highly effective strategy. Further improvement in such direction is expected by the introduction of miniaturized (i.e. 0.010 ") 
guidewires (and balloons) [15]. The first series of such devices is represented by the "Slender System" (Japan Lifeline) and 2 studies reported their use in the setting of CTO $[16,17]$.

\section{Limitations of the study}

This is a single center study reporting the results obtained by 2 highly experienced radial operators who selected the cases. Accordingly, the complexity of attempted CTO cannot be compared with other case series and the reported results may not be extended to operators with different radial experience.

Retrograde recanalization attempt was reserved for second attempts (with favorable anatomy) only so that the present paper does not allow any evaluation regarding the feasibility of TRA for retrograde approach for CTO PCI. Yet, some case reports $[18,19]$ and case series $[20,21]$ suggest that retrograde CTO techniques may be practiced by radial approach in patients with various anatomical complexity.

Finally, post-procedure renal function parameters were not systematically investigated so that the incidence of procedure-related contrast-induced nephropathy was not assessed.

\section{Conclusions}

The radial access constitutes a valuable option to treat patients undergoing CTO PCI by operators with high experience in TRA procedures. Procedure efficacy is mainly dependent on operator's experience with CTO techniques.

\section{Acknowledgements}

This paper is dedicated to the memory of Dr Hideo Tamai. His incredible technical skill and extraordinary dedication to patients cannot be forgotten by anybody who had the opportunity to see him working in his hospital.

\section{Conflict of interest: none declared}

\section{References}

1. Di Mario C, Werner GS, Sianos G et al. European perspective in the recanalisation of Chronic Total Occlusions (CTO): Consensus document from the EuroCTO Club. EuroIntervention, 2007; 3: $30-43$.

2. Burzotta F, Trani C, Mazzari M et al. Vascular complications and access crossover in 10,676 transradial percutaneous coronary procedures. Am Heart J, 2012; 163: 230-238.

3. Burzotta F, de Vita M, Trani C. Transradial approach for percutaneous coronary interventions on chronic total occlusions. Interv Cardiol, 2010; 2: 417-425.
4. Burzotta F, Mariani L, Trani $\mathrm{C}$ et al. Management and timing of access-site vascular complications occurring after trans-radial percutaneous coronary procedures. Int J Cardiol, 2013; 167: 1973-1978.

5. Kim JY, Lee SH, Choe HM, Yoo BS, Yoon J, Choe KH. The feasibility of percutaneous transradial coronary intervention for chronic total occlusion. Yonsei Med J, 2006; 31: 680-387.

6. Agostoni P, Biondi-Zoccai GG, de Benedictis Ml et al. Radial versus femoral approach for percutaneous coronary diagnostic and interventional procedures; Systematic overview and meta-analysis of randomized trials. J Am Coll Cardiol, 2004; 44: 349-356.

7. Jolly SS, Amlani S, Hamon M, Yusuf S, Mehta SR. Radial versus femoral access for coronary angiography or intervention and the impact on major bleeding and ischemic events: A systematic review and meta-analysis of randomized trials. Am Heart J, 2009; 157: 132-140.

8. Jolly SS, Yusuf S, Cairns J et al. Radial versus femoral access for coronary angiography and intervention in patients with acute coronary syndromes (RIVAL): A randomised, parallel group, multicentre trial. Lancet, 2011; 377: 1409-1420.

9. Galassi AR, Tomasello SD, Reifart N et al. In-hospital outcomes of percutaneous coronary intervention in patients with chronic total occlusion: Insights from the ERCTO (European Registry of Chronic Total Occlusion) registry. EuroIntervention, 2011; 7: 472-479.

10. Saito S, Tanaka S, Hiroe Y et al. Angioplasty for chronic total occlusion by using tapered-tip guidewires. Catheter Cardiovasc Interv, 2003; 59: 305-311.

11. RathoreS, HakeemA, Pauriah M, Roberts E, BeaumontA, MorrisJL. A comparison of the transradial and the trans-femoral approach in chronic total occlusion percutaneous coronary intervention. Catheter Cardiovasc Interv, 2009; 73: 883-887.

12. Yang $\mathrm{CH}$, Guo GB, Chen SM et al. Feasibility and safety of a transradial approach in intervention for chronic total occlusion of coronary arteries: A single-center experience. Chang Gung Med J, 2010; 33: 639-645

13. Liu W, Wagatsuma K, Toda M et al. Short- and long-term follow-up of percutaneous coronary intervention for chronic total occlusion through transradial approach: Tips for successful procedure from a single-center experience. J Interv Cardiol, 2011; 24: 137-143.

14. Burzotta F, De Vita M, Trani C. How to manage difficult anatomic conditions affecting transradial approach coronary procedures? Indian Heart J, 2010; 62: 238-244.

15. Masutani M, Yoshimachi F, Matsukage T, Ikari Y, Saito S. Use of slender catheters for transradial angiography and interventions. Indian Heart J, 2008; 60 (1 Suppl. A): A22-A26.

16. Matsukage T, Masutani M, Yoshimachi F et al. A prospective multicenter registry of 0.010 -inch guidewire and compatible system for chronic total occlusion: The PIKACHU registry. Catheter Cardiovasc Interv, 2010; 75: 1006-1012.

17. Matsukage T, Yoshimachi F, Masutani M et al. A new 0.010-inch guidewire and compatible balloon catheter system: The IKATEN registry. Catheter Cardiovasc Interv, 2009; 73: 605-610.

18. Taketani $Y$, Kaneda H, Saito S. Successful coronary intervention for chronic total occlusion using a retrograde approach with biradial arteries. J Invasive Cardiol, 2007; 19: E281-E284.

19. Burzotta F, Trani C, Coroleu S. Retrograde recanalization of left main from saphenous vein graft supported by percutaneous Impella Recover LP 2.5 assist device. J Invasive Cardiol, 2009; 21: E147-E150.

20. Rinfret S, Joyal D, Nguyen CM et al. Retrograde recanalization of chronic total occlusions from the transradial approach; early Canadian experience. Catheter Cardiovasc Interv, 2011; 78: 366-374.

21. Wu CJ, Fang HY, Cheng CI et al. The safety and feasibility of bilateral radial approach in chronic total occlusion percutaneous coronary intervention. Int Heart J, 2011; 52: 131-138. 